Tropical Journal of Pharmaceutical Research October 2012; 11 (5): 729-737

(C) Pharmacotherapy Group,

Faculty of Pharmacy, University of Benin

Benin City, 300001 Nigeria.

All rights reserved.

Available online at http://www.tjpr.org

http://dx.doi.org/10.4314/tjpr.v11i5.5

Research Article

\title{
Enhanced Recovery and Identification of a Tryptamine-Related Antibiotic Produced by Intrasporangium N8 from KwaZulu-Natal, South Africa
}

\author{
Vincent I Okudoh ${ }^{*}$ and Frederick M Wallis \\ School of Life Science (Microbiology), University of KwaZulu-Natal, Pietermaritzburg, Scottsville 3209, South Africa.
}

\begin{abstract}
Purpose: To isolate and identify an antibiotic produced by a soil bacterium, Intrasporangium strain N8, with antibacterial activity against both Gram-positive and Gram-negative bacteria.

Methods: Fermentation followed by extraction using a three-solvent system (petroleum ether, acetone and ethyl acetate) and $\mathrm{pH}$ precipitation, successfully separated the antibiotic complex from the culture broth. Purification was carried out using flash column chromatography (FCC), thin-layer chromatography (TLC) and reverse phase high performance liquid chromatography (HPLC). The identities of the molecules were elucidated by gas chromatography-mass spectrometry (GC-MS) analysis.

Results: Three main components of the antibiotic were isolated and identified as 4-methyl-3-penten-2one, 4-hydroxy-4-methyl-2-pentanone and N-acetyltryptamine. Bioassay results showed activity against both mammalian and plant pathogenic bacteria including Pseudomonas fluorescens, Xanthomonas campestris pv campestris, Escherichia coli and Serratia marcescens. Pseudomonas fluorescens (MIC = $0.0625 \mu \mathrm{g} / \mathrm{ml}$ ) and Xanthomonas campestris pv campestris $(\mathrm{MIC}=0.0026 \mu \mathrm{g} / \mathrm{ml}$ ) represent the two plant pathogenic genera that are notoriously difficult to contain in the field.

Conclusions: Since the antibiotic isolated during this study showed activity against both mammalian and plant pathogenic bacteria, it is hoped that this work will encourage further investigation in this field. This antibiotic could become very useful as an agricultural bacteriocide against some resistant plant pathogens.
\end{abstract}

Keywords: Intrasporangium N8, Tryptamine, Antibiotic, Purification, Pathogenic bacteria.

*Corresponding author: Email: Okudoh@ukzn.ac.za, vinokudoh@yahoo.com; Tel: +27-72-2184495. 


\section{INTRODUCTION}

Natural microbial products are the origin of most of the antibiotics in the market today. However, research on antibiotics and natural products has declined significantly during the last decade as a consequence of many factors, chief among which are the lack of interest in the field due to strong competition from synthetic compounds as sources of drugs [1]. There is also the ever expanding problem of bacterial resistance [2]. Consequently, there is an alarming scarcity of new antibiotic classes in the pipelines of the pharmaceutical industry. The above notwithstanding, microbial natural products remain the most promising sources of novel antibiotics [3], although new approaches are required to improve the efficiency of the discovery process. The choice of best approach at the screening phase and the challenges faced during the isolation, purification and identification of the active compounds are critical factors to success in this area.

In our approach, we have recognized that antibiotic compounds are in two main forms a salt form which is soluble in hydrophilic solvents and a basic form which is insoluble in these solvents. Therefore, a careful choice of solvent system provides an efficient and faster route to direct extraction of antibiotic compounds from complex mixtures [4]. These compounds can be identified by combinations of very powerful analytical tools that take advantage of their individual strengths. The first combination is gas chromatographymass spectroscopy (GC-MS) followed by gas chromatography-Fourier transform infrared (GC-FITR) and the second, HPLC-MS. Researchers are obtaining more information about their samples at a faster rate than was previously possible [5].

In this study, our aim was to isolate and identify an antimicrobial substance produced by Intrasporangium strain N8 using GC-MS analysis. Gas chromatography-mass spectroscopy (GC-MS) is the single most important tool for the identification and quantification of volatile and semi-volatile compounds in complex mixtures [6]. This study represents a part of the global search for new antibiotic compounds as organisms with multiple drug resistance continue to be a threat to public health worldwide [7].

\section{EXPERIMENTAL}

\section{Microorganism}

Strain N8 was isolated from a barnyard soil in the KwaZulu-Natal midlands, South Africa, in a massive actinomycetes-screening programme that led to the isolation of approximately 2600 strains [8]. Stock cultures were kept on agar slants or stored frozen in glycerol at minus $80{ }^{\circ} \mathrm{C}$. Identification of $\mathrm{N} 8$ to genus level was carried out according to Bergey's Manual of Determinative Bacteriology [9] and involved morphological, physiological, and biochemical characterization [8].

\section{Fermentation}

A mature slant culture of N8 was inoculated into a $250 \mathrm{ml}$ Erlenmeyer flask containing $100 \mathrm{ml}$ of seed medium, viz: Nutrient broth (NB) [meat extract $1 \mathrm{~g} / \mathrm{L}$, yeast extract $2 \mathrm{~g} / \mathrm{L}$, peptone $5 \mathrm{~g} / \mathrm{L}$ and sodium chloride $8.0 \mathrm{~g} / \mathrm{L}$, $\mathrm{pH} 7.1$ after sterilization]. The culture was incubated on a rotary shaker $(250 \mathrm{rpm})$ at 30 ${ }^{\circ} \mathrm{C}$ for $48 \mathrm{~h}$. Ten millilitres of this seed culture were transferred into a $250 \mathrm{ml}$ flask containing $200 \mathrm{ml}$ of the same medium with $5.0 \mathrm{~g}$ glycerol (Panreac, Barcelona, Spain) added. Shake-flask fermentation was carried out at $30{ }^{\circ} \mathrm{C}$ for 10 days under agitation at $250 \mathrm{rpm}$. Antibiotic production was monitored every $12 \mathrm{~h}$ by bioassay which included measuring the diameter of inhibition zones on plates containing test organisms.

One hundred $250 \mathrm{ml}$ flasks each containing $100 \mathrm{ml}$ of medium were used to obtain a total of approximately $10 \mathrm{~L}$ of strain N8 culture broth. The culture was centrifuged for $15 \mathrm{~min}$ at $9000 \times \mathrm{g}$ and the supernatant filtered 
through Cameo 25 AS acetate membrane filters prior to analysis.

\section{Isolation of the antibiotic}

Three solvents namely, petroleum ether, acetone and ethyl acetate, were used for the recovery of the antibiotic from the broth. A ratio of 1:1 solvent: broth was used and the extraction procedure was repeated three times. The first solvent, petroleum ether (PE), was added to the cell-free broth, the mixture shaken thoroughly and left overnight to settle. Separation of the organic and aqueous layers was achieved by means of a separating funnel. The PE extract was tested for antimicrobial activity before being discarded. The second solvent, acetone, was added to the aqueous layer and the resultant precipitate removed by centrifugation at $9000 x g$ for 15 minutes. This precipitate too was tested for antimicrobial activity by the agar disk-diffusion method [10] following dissolution in methanol $(10 \mathrm{ml})$. The remaining solution was concentrated 10 -fold with a Buchi rotary evaporator at $30^{\circ} \mathrm{C}$. Finally, the solution was extracted with an equal volume of ethyl acetate (1:1)

The aqueous layer was further extracted by adjusting the $\mathrm{pH}$ to 3 and 8.2 with $2 \mathrm{M} \mathrm{HCL}$ and $2 \mathrm{M} \mathrm{NaOH}$ respectively, and allowing the mixture to crystallize overnight at $4^{\circ} \mathrm{C}$. The crystals were recovered by filtering through Whatman's glass microfiber and dried overnight at ambient temperature. Two bioassay methods, agar well- and agar diskdiffusion, were used to monitor the antimicrobial activity of the extracts. These procedures allowed efficient extraction of the antibiotic complex from the culture broth.

\section{Purification by chromatography}

Having established that the antibacterial agent resided in the ethyl acetate extract, various chromatographic techniques were used to further purify it. Flash column chromatography (FCC) followed by thin layer chromatography (TLC) was carried out on each solvent extract using coated Merck Kieselgel $60 \mathrm{~F}_{254}$ plastic sheets. Thin layer chromatography plates were visualized by first checking for fluorescence under ultraviolet (UV) light at $254 \mathrm{~nm}$ followed by staining with anisaldehyde before drying with a heat gun. The active material was recovered from the TLC plates by excising UV fluorescent bands from the chromatogram, packing the material into a Pasteur pipette and eluting through glass wool using ethyl acetate as eluate. All the preparations were further concentrated in a Buchi Rotavapor EL 130 at $30{ }^{\circ} \mathrm{C}$ before testing for biological activity. A further purification step was also carried out on each solvent extract using the Harrison research chromatotron technique. Care was taken not to increase the concentration of methanol beyond $50 \%$ because of its tendency to dissolve the silica gel on the chromatotron plates. Fractions collected at $1 \mathrm{~min}$ intervals were pooled, and spotted onto TLC plates. The positions of the spots were detected under UV light or by staining with anisaldehyde. Corresponding positions on duplicate plates were excised, eluted and tested for antimicrobial activity.

Further purification was carried out by reverse phase high performance liquid chromatography (HPLC) and isocratic HPLC analysis was conducted on the ethyl acetate extract using a Data Apex Clarity chromatography station equipped with an Alltech $\mathrm{C}-18$ reverse phase column and monitored by absorbance at $280 \mathrm{~nm}$. Separations using a gradient system were performed on a Varian Analytical Instruments HPLC equipped with a Thermo Separation Products automated fraction collector. Fifty microlitres of the extract were injected into a Hypersil 5 ODS analytical column $(25 \mathrm{~cm} x$ $10 \mathrm{~mm}$ ) followed by gradient elution in decreasing concentrations of methanol $(80 \%$ - 50\%) in water. Absorbance was measured using a UV detector (UV6000LP, Thermo Finnigan) at $280 \mathrm{~nm}$. 


\section{Identification of the antibiotic}

Gas chromatography-mass spectroscopic (GC-MS) analysis of the compound showing antibiotic activity was carried out. Mass spectra were recorded on a ThermoFinnigan Polaris/ QCQ Plus instrument coupled to a Varian CP-3800 gas chromatograph. Low and high resolution mass spectra were measured on a Kratos MS 80 RF double focusing magnetic sector instrument at 70 eV. Spectra were obtained using both an electron impact mode (EI) and a chemical ionization mode $(\mathrm{Cl})$. The MS profile of the antimicrobial substance produced by strain N8 was compared to MS profiles from the databases of known natural compounds [11].

\section{Bioassay}

The antimicrobial activity of the active compound was determined in vitro by agar disk diffusion or micro-dilution methods in terms of minimum inhibitory concentration (MIC) according to the protocol recommended by the Clinical and Laboratory Standards Institute (CLSI) [formerly National committee for Clinical Laboratory Standards (NCCLS) [10]. The test organisms used were Escherichia coli, Staphylococcus aureus, Pseudomonas fluorescens, Serratia marcescens, Enteroococcus faecalis, Candida utilis and Xanthomonas campestris pv campestris, all from the culture collection in the Department of Microbiology, University of KwaZulu-Natal (UKZN), South Africa. The above organisms were selected on the basis of a 1999 survey of major human and plant pathogens prevalent in KwaZulu-Natal hospitals and the University vegetable garden. All test organisms were stored on thick Nutrient agar (NA) at $\pm 4^{\circ} \mathrm{C}$ until required.

\section{Statistical analysis}

A one-way analysis of variance model was used to compare the average inhibition zone diameter (AIZD) recorded for the seven test organisms in a triplicate experiment.
Statistical analysis of the data was carried out using SPSS (version 11.5). Mean and standard deviation were computed for each of the organisms. The differences were noted on the basis of an F-test, and the corresponding $p$-value, at a $5 \%$ level of significance. Fisher's least significant difference method was used to determine the individual mean difference between the organisms.

\section{RESULTS}

An earlier report on strain N8 using morphological, physiological and biochemical characterization identified the organism as an Intrasporangium species (Fig 1) [8]. During fermentation, maximum yield of the antibiotic was obtained after 7 days incubation. Optimum antibiotic yield was obtained at neutral $\mathrm{pH}$ and an incubation temperature of $30{ }^{\circ} \mathrm{C}$.

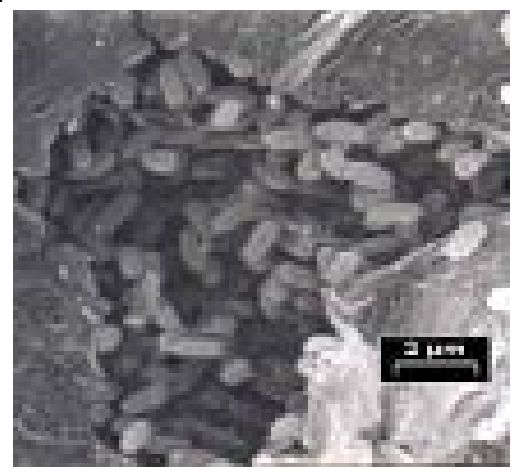

Fig 1: Scanning electron micrograph of Intrasporangium strain N8 mycelium (arrow shows oval shaped spores exposed inside a broken vesicle).

The use of three solvents (petroleum ether, acetone and ethyl acetate) during extraction, together with $\mathrm{pH}$ precipitation, successfully isolated the antibiotic from the broth. Separation of the organic and aqueous layers was easily achieved using a separating funnel. The organic layer when dried with anhydrous $\mathrm{NaCl}$ and evaporated to near dryness produced a yellow liquid with some yellow crystals present on the sides of the flask after cooling to $25^{\circ} \mathrm{C}$. Bioassay results 


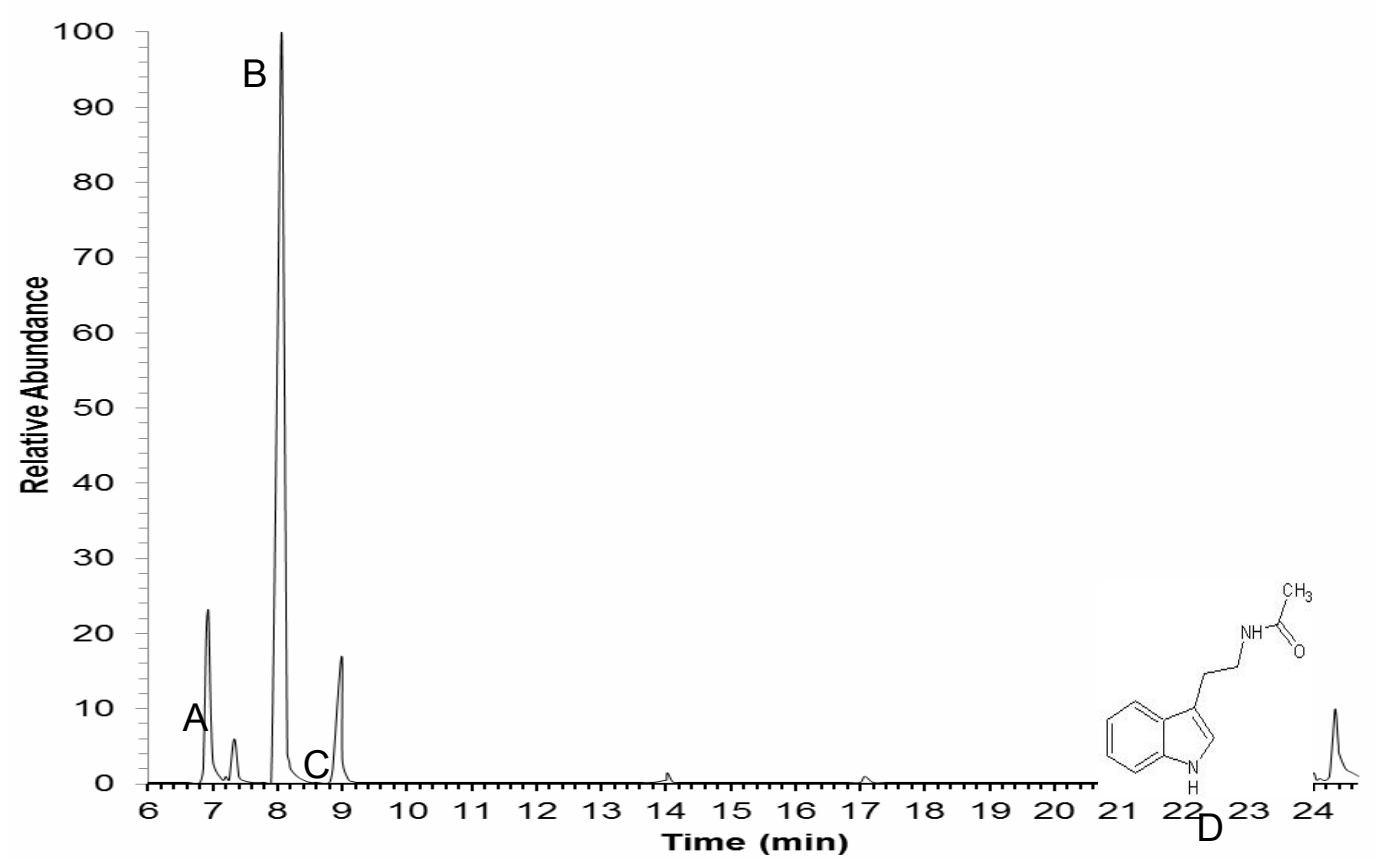

Fig 2: Gas chromatogram of the antimicrobial substance in the ethyl acetate extract after extraction of the culture fluid of strain N8. Components A - D were subjected to mass spectroscopy analysis. Note the occurrence of a possible tryptamine structure (D) which, is essential for antimicrobial activity $[15,16]$.

for the three solvent extracts showed clear inhibition of Escherichia coli with clear zones around the paper disks dipped in the ethyl acetate extract (EAF). Large inhibition zones (30 $\mathrm{mm}$ diameter) were also observed for the acetone extract $(\mathrm{AH})$, but these were cloudy, unlike the very clear zones produced by the EAF ( $21 \mathrm{~mm}$ diameter). No zone of inhibition occurred with the petroleum ether extract (PE).

Gas chromatography-mass spectroscopic (GC-MS) data analysis of the EAF indicated that the antibiotic comprised four main components labeled A - D (Fig 2). These components separated by gas chromatography (GC) were then subjected to mass spectrum analysis and their retention indices compared to computer database listings of known compounds (11).

The compounds detected by GC-MS had their molecular formulae elucidated as:
$\mathrm{C}_{6} \mathrm{H}_{10} \mathrm{O}$ ( $\mathrm{A}-\mathrm{B}=$ 4-methyl-3-penten-2-one); $\mathrm{C}_{6} \mathrm{H}_{12} \mathrm{O}_{2} \quad(\mathrm{C}=$ 4-hydroxy-4-methyl-2-pentanone); and $\mathrm{C}_{12} \mathrm{H}_{14} \mathrm{~N}_{2} \mathrm{O}$ (D = N-[2-(1H-indol-3$\mathrm{yl}$ )ethylacetamide), as shown in Table 1. The closest matching structure from the computer database of known compounds was found for $\mathrm{C}_{12} \mathrm{H}_{14} \mathrm{~N}_{2} \mathrm{O}(\mathrm{D})$ with $71 \%$ probability.

Analyses of the GC-MS data suggested that components $A$ and $B$ are similar compounds since they have the same molecular weight and formula. Their different retention indices strongly suggest that they are probably isomers. Thus a total of three main compounds were detected by GC. In GC application, the lighter molecules elute first followed by the heavier ones [12]. Therefore, of the main compounds separated by the GC, component $\mathrm{D}$ was the heaviest molecule since it eluted last from the column. It has an MS spectrum that closely resembles $(71 \%)$ that of an indolethylacetamide $(=\mathrm{N}$ acetyltryptamine) as shown in Figure 3. 
Table 1: Compounds identified* by mass spectroscopic data analysis following gas chromatographic separation of the antibiotic produced by strain N8

\begin{tabular}{|c|c|c|c|c|c|}
\hline $\begin{array}{l}\text { Component } \\
\text { of antibiotic } \\
\text { molecule }\end{array}$ & $\begin{array}{l}\text { Retention } \\
\text { time (min) }\end{array}$ & $\begin{array}{l}\text { Mol. } \\
\text { weight }\end{array}$ & $\begin{array}{l}\text { Mol. } \\
\text { formula }\end{array}$ & Index name of compound & $\begin{array}{c}\text { Prob. } \\
(\%)\end{array}$ \\
\hline $\bar{A}$ & 6.93 & 98 & $\mathrm{C}_{6} \mathrm{H}_{10} \mathrm{O}$ & 4 methyl-4-penten-2-one & 37 \\
\hline B & 8.06 & 98 & $\mathrm{C}_{6} \mathrm{H}_{10} \mathrm{O}$ & - 4 methyl-3-penten-2-one & 39 \\
\hline C & 8.99 & 116 & $\mathrm{C}_{6} \mathrm{H}_{12} \mathrm{O}_{2}$ & - 4-hydroxy-4-methyl-2-pentanone & 57 \\
\hline D & 24.30 & 202 & $\mathrm{C}_{12} \mathrm{H}_{14} \mathrm{~N}_{2} \mathrm{O}$ & - $\quad \mathrm{N}$-[2-(1H-indol-3-yl)ethyl acetamide & 71 \\
\hline
\end{tabular}

*Identification based on the built-in computer database of known compounds [11].
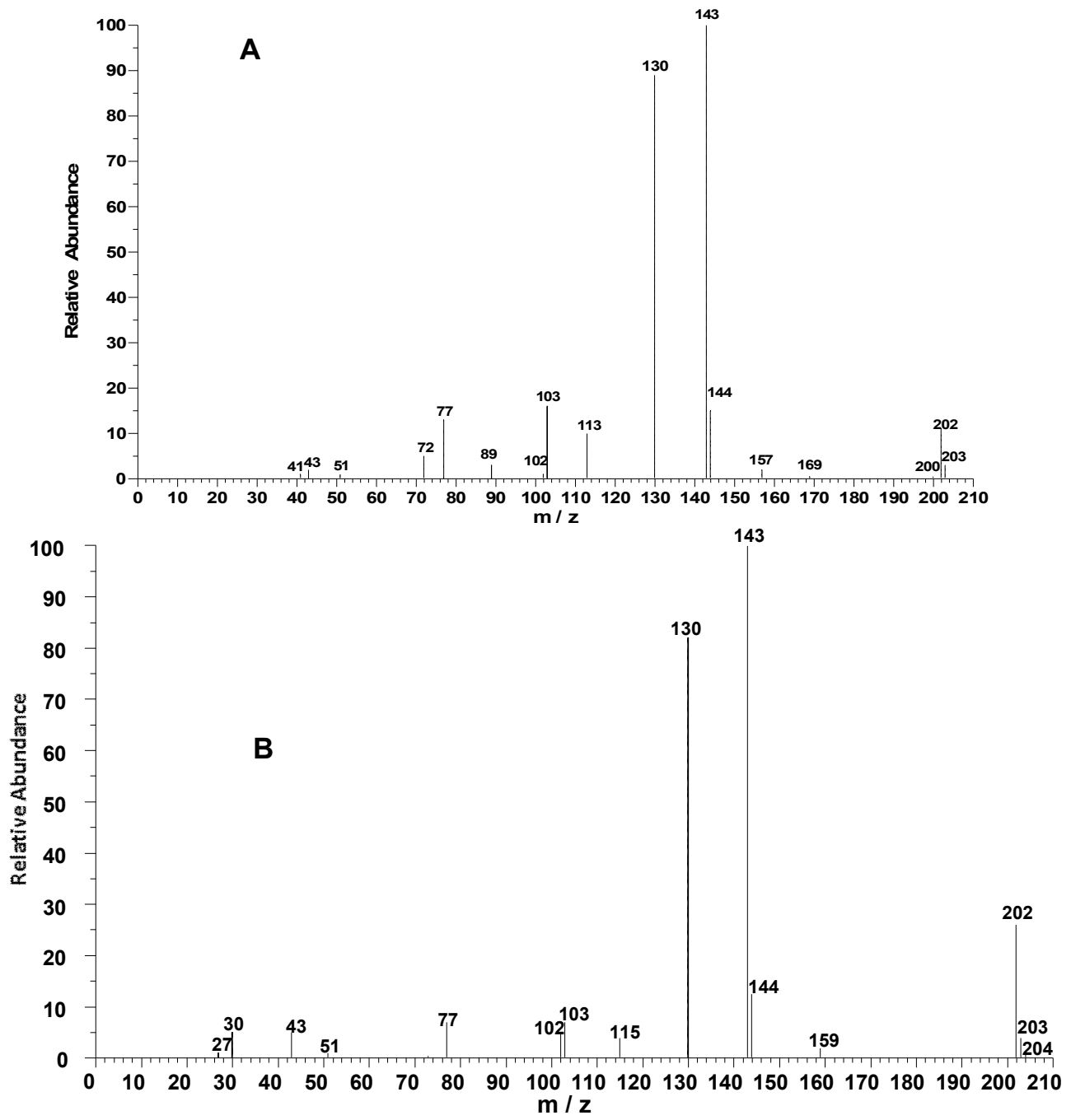

Fig 3: Structural identity of component $D$ : (A) full mass spectrum of component $D$; (B) matching (71 \%) full mass spectrum of $\mathrm{N}-(2-(1 \mathrm{H}$-indol-3-yl)ethylacetamide (=N-acetyltryptamine).

The $71 \%$ probability reflects how closely the spectrum of the compound matched the index value obtained from a chemical library (computer database) of known natural compounds [11]. 
Table 2: Antimicrobial activity of the antibiotic produced by Intrasporangium strain N8 against test organisms

\begin{tabular}{|c|c|c|c|}
\hline Test organism & $\begin{array}{l}\text { Extent of } \\
\text { inhibition }\end{array}$ & $\begin{array}{l}\text { Mean inhibition zone } \\
\text { diameter }(\mathrm{mm})^{*}\end{array}$ & $\begin{array}{c}\text { Minimum inhibitory } \\
\text { concentration }(\mu \mathrm{g} / \mathrm{ml})^{\wedge}\end{array}$ \\
\hline Staphylococcus aureus & + & $10.0 \pm 1.00$ & $0.0078 \pm 0.000$ \\
\hline Escherichia coli & +++ & $25.0 \pm 0.58$ & $0.0039 \pm 0.000$ \\
\hline Streptococcus faecalis & ++ & $20.0 \pm 0.00$ & $0.0625 \pm 0.000$ \\
\hline Serratia marcescens & ++ & $15.0 \pm 1.16$ & $0.1250 \pm 0.000$ \\
\hline Pseudomonas fluorescens & ++++ & $26.0 \pm 1.00$ & $0.2500 \pm 0.000$ \\
\hline Candida utilis & ++ & $21.0 \pm 1.53$ & $0.0020 \pm 0.000$ \\
\hline $\begin{array}{l}\text { Xanthomonas campestris } p v \\
\text { campestris }\end{array}$ & ++++ & $28.0 \pm 0.58$ & $0.0026 \pm 0.001$ \\
\hline
\end{tabular}

$L S D$

2.652

0.00116

* Screening on Mueller Hinton (MH) agar, ^screening in $\mathrm{MH}$ broth; + = positive inhibition (3-10 mm); ++ = 11-20mm; $+++=21-25 \mathrm{~mm} ;++++=26-30 \mathrm{~mm}$

Further bioassay experiments showed that component $D$ was responsible for the observed antibiotic activity. It showed good antimicrobial activity against all the test organisms used, especially against Pseudomonas fluorescens and Xanthomonas campestris pv campestris, with MICs of $0.0625 \mu \mathrm{g} / \mathrm{ml}$ and $0.0026 \mu \mathrm{g} / \mathrm{ml}$, respectively. Its spectrum of activity included both gramnegative and gram-positive bacteria and the yeast Candida utilis (Table 2).

We observed a significant difference in average inhibition zone diameters between the test organisms, $F(6,14)=130.351, p<$ 0.001 . At $5 \%$ level, the mean AIZD for Xanthomonas campestris pv campestris was significantly greater than that for all the other organisms except Pseudomonas fluorescens. All other AIZD were significantly different except for Candida utilis and Streptococcus faecalis, $\mathrm{t}(14)=0.667, p=0.405$ and Escherichia coli and Pseudomonas fluorescens, $\mathrm{t}(14)=-1.33, p=0.108$.

\section{DISCUSSION}

Most secondary metabolites, including antibiotics, are usually produced at a very late stage during the growth of an organism [13]. On this assumption, antibiotic production in the growth medium was monitored every $12 \mathrm{~h}$ over the fermentation period to establish the time of maximum yield. The growth curve of strain N8 indicates that the organism should be grown for 7 to 10 days in a simple medium before harvesting the broth for antibiotic analysis. In this study, harvesting of the secondary metabolite was done on the tenth day, allowing time for the stationary phase to be reached at which time a higher yield was available.

The solvent extraction procedure used enhanced the separation of compounds of different polarities. Hydrophilic organic substances in the broth were targeted and extracted with polar solvents while the hydrophobic ones were solubilized in the non-polar solvents used. The non-polar solvent, petroleum ether, was used to remove lipids from the broth. Acetone precipitation helped to remove most solutes, especially protein, from the broth and ethyl acetate was used for extracting the remaining polar organic substances. The extraction profile suggested that the antibacterial agent produced by strain N8, is a polar organic compound because of its solubility in polar organic solvents, and not a lipid due to its insolubility in the non-polar solvent petroleum ether. The polar nature of the active substance was confirmed when the petroleum ether extract failed to show any biological activity against $E$. coli. This preliminary result was an important step in this investigation as the majority of antibiotics are polar [14].

Trop J Pharm Res, October 2012;11(5): 735 
The successful extraction of the antimicrobial agent from the broth with ethyl acetate and other polar solvents led us to focus on the ethyl acetate fraction (EAF) in subsequent work. We found important issues to be choice of growth media; solvents used; and a continuous monitoring of biological activity. Stringent controls are essential during bioassays and care must be taken to avoid erroneous results; for example freeze drying samples before bioassay may concentrate various constituents of the culture medium giving rise to false positive results. Also the media used for bioassay must be able to support vigorous growth of the test organism in the absence of potentially inhibitory compounds. If possible, complex media should be avoided; the simpler the medium the better.

Attempts were made to assign a chemical structure to the antibiotic produced by strain N8 using all the data available. The indications are that it is a tryptamine, the chemical structure of which is postulated to be as shown in Fig 4.

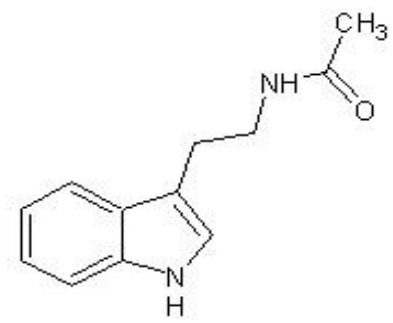

Fig 4: Chemical structure of antibiotic produced by Intrasporangium strain N8

$\mathrm{N}$-acetyltryptamine is reported to be produced by Thermoactinomyces strain TA66-2 [15] and Streptomyces species strain TN58 [16]. These authors also reported that thiazole was produced simultaneously with $\mathrm{N}$ acetyltryptamine. Tryptamine is a monoamine alkaloid found in plants, fungi, and animals and the backbone structure of a group of compounds known collectively as tryptamines [17]. This group includes many biologically active compounds, including neurotransmitters and psychedelic drugs [18].
Tryptamine is also found in trace amounts in the brains of mammals and is believed to play a role as a neuromodulator or neurotransmitter. Structurally, it is based on the indole ring, and is chemically related to the amino acid tryptophan, from which its name is derived [17]. A closely related structure to tryptamine is melatonin (Fig 5) which has been associated with the ability to inhibit tumour growth in vivo and in vitro.<smiles>COc1ccc2[nH]cc(CCNC(C)=O)c2c1</smiles>

Fig 5: Chemical structure of melatonin

Some tryptamine-based antibiotics have also been reported. An example is, nematophin (3-indoleethyl (3'-methyl-2'-oxo)pentanemide), isolated from Xenorhabdus nematophilus[[19]. The natural compound showed strong in vitro bioactivity against a series of fungal and bacterial species [19]. Other derivatives of this compound, including alkyl- and aryl-substitutions at the 1-position, gave elevated antimicrobial activity [20]. The same study found that the amide $(\mathrm{NH})$ group in the nematophin structure is essential for bioactivity. In another published study, it was reported that the 2-phenyl derivative of nematophin exhibited exceptional activity against methicillin-resistant Staphylococcus aureus, whereas the isosteric benzimidazole analogue was much less active [21].

\section{CONCLUSION}

The antibiotic produced by Intrasporangium strain N8 actively inhibited the growth of all the test organisms used, especially that of Pseudomonas fluorescens and Xanthomonas campestris pv campestris, with MIC of 0.0625 and $0.0026 \mu \mathrm{g} / \mathrm{ml}$, respectively. Members of these plant pathogenic genera are difficult to contain in the field and N8 might thus prove useful as a biocontrol agent. Our results showed that strain N8 produces an antibiotic that is readily soluble in acetone, ethyl acetate and methanol, but insoluble in 
petroleum ether. It is a polar compound, very stable in water and could thus be easily formulated as an agricultural bactericide. It is also heat stable and passed undamaged through the gas chromatograph. An important feature of the compound is the presence of a tryptamine related structural component which is essential for antimicrobial activity. It is postulated that the antibiotic produced by Intrasporangium strain N8 has the same or a similar mechanism of action to tryptamine.

\section{ACKNOWLEDGEMENT}

This work was supported by the National Research Foundation (NRF) of South Africa. We thank the following staff of the University of KwaZulu-Natal for their help, guidance and support: Professors Mark Laing (financial support) and Fanie van Heerden (assistance with chemical aspects of the work); Marnie Light for HPLC analysis; Dr Collin Southway for GCMS analysis; Marylin Christian, Diane Fowlds, Igrid Schlosser and Kerry Lindsey for supplying the test organisms; Natalie Jones and Prof Stefan Schmidt for administrative advice and encouragement, respectively.

\section{REFERENCES}

1. Pelaez F. The historical delivery of antibiotics from microbial natural products-can history repeat? Biochem Pharmacol 2006; 71(7): 981990.

2. Chopra I, Hodgson J, Metcalf B, Poste G. The search for antimicrobial agents effective against bacteria resistant to multiple antibiotics. Antimicrob Agents Chemother 1997; 41: 497-503.

3. Wang J, Soisson SM, Young $K$, Shoop W, Kodali $S$, Galgoci A, Painter $R$, Parthasarathy $G$, Tang YS, Cummings $R$, et al. Platensimycin is a selective FabF inhibitor with potent antibiotic properties. Nature 2006; 441: 358-361.

4. Brites Alves AM, Morao A, Cardoso JP. Isolation of antibiotics from industrial fermentation broths using membrane technology. Desalination 2002; 148(Pt 1-3): 181-187.

5. Joshi S. HPLC separation of antibiotics present in formulated and unformulated samples. $J$ Pharm Biomed Anal 2002; 28(5): 795-809.

6. Santos FJ, Galceran MT. The application of gas chromatography to environmental analysis. Trends Anal Chem 2002; 21: 672-685.
7. Cohen ML. Changing patterns of infectious disease. Nature 2000; 406: 762-767.

8. Okudoh VI, Wallis FM. Antimicrobial activity of rare actinomycetes isolated from natural habitats in KwaZulu-Natal, South Africa. S Afr J Sci 2007; 103: 216-222.

9. Williams ST, Sharpe ME, Holt TG. Bergey's manual of systematic bacteriology. Williams and Wilkins, Baltimore, 1989.

10. Clinical and Laboratory Standards Institute (CLSI) M7-A Methods for Dilution Antimicrobial Susceptibility Tests for Bacteria that Grow Aerobically; Approved Standard, 7th ed. Villanova, $\mathrm{Pa}, 2004$.

11. National Institute of Standards and Technology (NIST). Standard reference database number 69. In: Chemistry Web Book, Linstrom PJ, Mallard WG, editors. National Institute of Standards and Technology [Internet]. Gaithersburg MD, 208992008 [cited 2008 Dec 15]. Available from www. nist.gov.

12. Krupp EM, Donard OFX. Isotope ratios on transient signals with GC-MC-ICP-MS. Int J Mass Spectrom 2005; 242(2-3): 233-242.

13. Barrios-Gonzalez J, Fernandez FJ, Tomasini A, Mejia A. Secondary metabolites production by solid-state fermentation. Malaysian J Microbiol 2005; 1: 1-6.

14. Lancini G, Lorenzetti R. Biotechnology of antibiotics and other bioactive microbial metabolites. Plenum Press, New York, 1993. pp 49-57.

15. Korkmaz CA, Hames-Kocabas EE, Uzel A, Bedir E. Tryptamine-derived amides with thiazole ring system from Thermoactinomyces strain TA66-2. Magn Reson Chem 2008; 46: 80-83.

16. Ben Ameur-Mehdi $R$, Shaaban KA, Rebai IK, Smaoui S, Bejar S, Mellouli L. Five naturally bioactive molecules including two rhamnopyranoside derivatives isolated from the Streptomyces sp. strain TN58. Nat Prod Res 2009; 23: 1095-1107.

17. Wurst M, Kysilka R, Flieger M. Psychoactive tryptamines from basidiomycetes. Folia Microbiol 2002; 47(1): 3-27.

18. Jones RS. Tryptamine: a neuromodulator or neurotransmitter in mammalian brain? Prog Neurobiol 1982; 19: 117-139.

19. Li J, Chen G, Webster JM. Nematophin, a novel antimicrobial substance produced by Xenorhabdus nematophilus (Enterobactereaceae). Can J Microbiol 1997; 43: 770-773.

20. Himmler T, Pirro F, Schneer N. Synthesis and antibacterial in vitro activity of novel analogues of nematophin. Bioorg Med Chem Lett 1998; 8: 2045-2050.

21. Kenneday G, VizianoPirro $M$, Winders JA, Cavallini $P$, Gevi M, Micheli F, Rodegher $P$, Seneci $P$, et al. Studies of the novel anti-staphylococcal compound nematophin. Bioorg Med Chem Lett 2000; 10: 1751-1754. 